\title{
FROM ORTHOGRAPHY TO MEANING: AN ELECTROPHYSIOLOGICAL INVESTIGATION OF THE ROLE OF PHONOLOGY IN ACCESSING MEANING OF CHINESE SINGLE-CHARACTER WORDS
}

\author{
K. WANG, ${ }^{a, b, c, d}$ A. MECKLINGER, ${ }^{c *}$ J. HOFMANN ${ }^{c}$ AND \\ X. WENG ${ }^{a}$ \\ a Laboratory of Higher Brain Functions, Institute of Psychology, Chi- \\ nese Academy of Sciences, Beijing, China \\ ${ }^{b}$ Neuropsychology and Applied Cognitive Neuroscience Laboratory, \\ Key Laboratory of Mental Health, Institute of Psychology, Chinese \\ Academy of Sciences, Beijing, China \\ 'Department of Psychology, Experimental Neuropsychology Unit, \\ Saarland University, Saarbrücken, Germany \\ ${ }^{d}$ Graduate School, Chinese Academy of Sciences, Beijing, China
}

\begin{abstract}
Homophone interference effects in Stroop experiments are often taken as evidence for the hypothesis that semantic access in written Chinese language is mediated by activation of phonological processing. We here aim to test this hypothesis with Chinese single-character words by means of event related potential (ERP) recordings. Using color words, homophones of color words and color-word associates as materials in a Stroop task, we found behavioral Stroop interference effects for all stimulus types and an N450 for incongruent color words and color-word associates. Critically, there was no difference in the ERP waveforms elicited by congruent and incongruent homophones in the N450 time window. However, in a later time window $(600-800 \mathrm{~ms})$ the incongruent homophones elicited an apparent positivity over left posterior regions. A similar effect was also observed for incongruent color words. These findings thus indicate that phonology does not play an important role in semantic activation of Chinese single-character words, and that the behavioral Stroop effects for homophones possibly arises at later stage of lexical processing. (C) 2010 IBRO. Published by Elsevier Ltd. All rights reserved.
\end{abstract}

Key words: semantic, phonology, N450, Stroop, homophone, ERP.

The influential dual-route cascaded model of reading assumes that semantic access is accomplished either directly via orthography or indirectly via phonology (Coltheart et al., 2001). While it is generally agreed that phonology plays an important role in semantic access in alphabetic languages nowadays (Frost, 1998), the relative importance of phonology in retrieving meaning of a visual Chinese word is still a matter of debate. Since the mapping between orthography and phonology is not transparent, it is often suggested that indirect semantic access via phonology is not efficient in Chinese (Wong and Chen, 1999).

*Corresponding author. Tel: $++49-681-302-6515$; fax: $++49-681-$ 302-6516.

E-mail address: mecklinger@mx.uni-saarland.de (A. Mecklinger). Abbreviations: ANOVA, analysis of variance; EEG, electroencephalogram; ERP, event related potential; ERPs, event related potentials.
While a number of studies do provide support for this direct access hypothesis (Chen and Shu, 2001; Meng et al., 2008), many behavioral experiments indicate that phonology plays an important role in accessing meaning of Chinese words (Chen and Tsoi, 1990; Guo et al., 2005; Perfetti and Zhang, 1995; Saalbach and Stern, 2004; Spinks et al., 2000; Tan and Perfetti, 1997; Xu et al., 1999), such as in Stroop tasks (Chen and Tsoi, 1990; Guo et al., 2005; Saalbach and Stern, 2004; Spinks et al., 2000).

In classical Stroop tasks participants are asked to respond to the ink or back-color of words and to ignore the word meaning (Stroop, 1935). Longer reaction times for incongruent trials ("red" printed in green ink or embedded in green back-color) compared to congruent trials ("red" in red or embedded in red back-color) or neutral words were repeatedly observed. Homophones of color words (e.g., 洪,/hong2/,mean flood, homophone of "red" in Chinese, the numeral here refers to Chinese tone) are often used to investigate the role of phonology in semantic access. For example, some studies found that semantically incongruent homophones of color words (e.g., 洪 in green) elicited significantly longer reaction times and/or higher error rates than congruent homophones (e.g., 洪 in red) (Chen and Tsoi, 1990; Guo et al., 2005; Saalbach and Stern, 2004; Spinks et al., 2000). Since the meaning of homophones is semantically unrelated to colors, this effect is typically interpreted as such that homophones activated the meaning of color words via the shared phonological code (Chen and Tsoi, 1990; Guo et al., 2005; Saalbach and Stern, 2004; Spinks et al., 2000).

\section{HOMOPHONE INTERFERENCE EFFECTS AND THE DUAL-ROUTE MODEL OF READING}

However, this semantic-based explanation for the homophone Stroop interference effect should be interpreted cautiously. Within the framework of the dual-route model it has been suggested that at least two processes were involved when participants perform Stroop tasks, namely a semantic and a phonological process (Coltheart et al., 1999; Saalbach and Stern, 2004). Words with strong semantic association to colors, such as GRASS, may produce a Stroop interference effect relative to neutral words such as HEART (Klein, 1964; Saalbach and Stern, 2004); this effect is taken to be semantic in nature and can be attributed to a conflict in the semantic lexicon. Additionally, reaction times for naming the color of pronounceable nonwords, such as ITS, may be greater than naming the color 
of random consonant strings (Bakan and Alperson, 1967); this effect is assumed to be phonological in nature.

In the same vein, the behavioral homophone effects in Stroop tasks could be attributed to the conflict in the semantic lexicon between the meanings of colors and the closely related meaning of color words activated by the identical pronunciation of homophones, as argued by the previous researchers (Chen and Tsoi, 1990; Guo et al., 2005; Saalbach and Stern, 2004; Spinks et al., 2000). However, the effects could also occur because of the conflicting phonologies of color name and written word. With behavioral indices, such as reaction times and error rates, it is hard (but also possible, see the study of Saalbach and Stern (2004)) to distinguish semantic and phonological components in the Stroop effects. However, event related potentials (ERP) due to their excellent temporal resolution in the millisecond domain are a valuable tool to investigate the semantic activation processes and to disentangle the contribution of the semantic component and the phonological component to the Stroop effects.

Two ERP components have been reported in Stroop tasks (Liotti et al., 2000). It has been suggested that a negativity at about 400 to $500 \mathrm{~ms}$ post stimulus that is larger for incongruent than for congruent color words (hereafter N450) is a reliable ERP index of semantic conflict between the meaning of color words and the response to colors. Thus, the N450 may reflect the semantic component in the behavioral Stroop effects. A second ERP component found in Stroop tasks is a late positivity peaking about 600-700 ms post-stimulus onset that is centered over left posterior superior scalp and elicited by incongruent color words. Given that the late positivity overlapped with response times in Stroop tasks and was most pronounced at recording sites in close proximity to brain regions involved in processing of word meaning it is conceivable that this component may reflect the processing of word meaning in case of color/word incongruency at a late processing stage.

\section{PURPOSE OF THIS STUDY}

The main purpose of this study was to further explore the role of phonology in semantic access in Chinese by means of ERP recordings. It has been suggested that the N450 is a reliable ERP index of semantic conflict between the meaning of color words and the response to colors. If homophones induce behavioral Stroop effects in Chinese languages by activating the meaning of the color word, then we could expect to observe similar N450 effects for incongruent homophones and color words. Thus, the basic assumption of this study is that if an N450 is observed for both incongruent homophones and color words, then phonology plays a role in semantic access. Conversely, if no N450 is elicited by homophones, then the phonology does not have an important role in Chinese semantic access.

We also predicted that there should be a late and posteriorly distributed positivity for incongruent homophones and for incongruent color words, because both classes of items have related-but-incongruent phonology with the colors and this may lead to the additional processing of word meaning.

In addition, we also aimed at exploring the sensitivity of the N450 to the amount of semantic conflict in the Stoop paradigm. To this end, one more stimulus type was added, the color-word associates. Items like "grass" have high semantic associations with certain colors (green here). Behavioral Stroop effects for these items have been observed, though smaller than those induced by incongruent color words (Klein, 1964; Saalbach and Stern, 2004). If the $\mathrm{N} 450$ is related to the amount of semantic conflict, then we expected to observe an $\mathrm{N} 450$ also for incongruent colorword associates, but due to their lower semantic association strength the N450 for incongruent color-word associates should be smaller or topographically more circumscribed than the one for incongruent color words.

\section{EXPERIMENTAL PROCEDURES}

\section{Participants}

Nineteen healthy native Chinese speakers (mean age $=26$, range $=24-33$, nine female), who finished at least 14 years of education in China and studied in Germany when the experiment was performed, provided informed consent to participate in the study in return for 8 Euro per hour. All of them were right-handed and had normal or corrected-to-normal vision. Data of two additional participants were discarded due to recording failures or a large number of artifacts in their ERP data.

\section{Stimuli}

Consistent with many previous studies, red (红,/hong2/), green (绿,/lü4/), yellow (黄,/huang2/), and blue (蓝,/lan2/) were used as four colors. For the selection of the color-word associates, a questionnaire was sent to 18 Chinese adults. They were encouraged to give a one-character noun which was most associated with each of four colors. Items which were most frequently listed were used as color-word associates: fire (火,/huo3/), grass (草 ,/cao3/), orange (橙,/cheng2/), and sea (海,/hai3/). Homophones are frequently used words which share the same pronunciations and tones with corresponding color words, and have no orthographic similarity to and semantic association with color words. They are flood (洪/hong2/), law (律//ü4/), emperor (皇/ huang2/) and fence (栏, (lan2/).

Neutral words together with a high proportion of color patches $(24.8 \%)$ were taken as filler materials. None of the neutral words had orthographical, phonological or semantic relationship with other words materials in this experiment. Word complexity, such as structure and strokes, was also matched when choosing neutral words. Color patches were rectangles in 65 pixel $\times 73$ pixel. Each word stimulus was presented in size of 46 pt in black with font Kai, and was embedded into a colored rectangle.

Each incongruent item (such as "green" in a red rectangle) was repeated 10 times; each congruent item was repeated 30 times. In total 1128 trials were composed from 120 incongruent color words, 120 congruent color words, 120 incongruent colorword associates, 120 congruent color-word associates, 120 incongruent homophones, 120 congruent homophones, 128 neutral words, and 280 color patches.

\section{Electrophysiological methods}

Scalp electroencephalogram (EEG) was recorded from an array of 59 silver/silver-chloride electrodes embedded in an elastic cap (EasyCap $\mathrm{GmbH}$, Herrsching-Breitbrunn, Germany), and ampli- 
fied from $\mathrm{DC}$ to $100 \mathrm{~Hz}$ at a sampling rate of $500 \mathrm{~Hz}$. The EEG was acquired referenced to the left mastoid and re-referenced off-line to linked mastoids. Further off-line data processing included a digital low-pass filter set to $30 \mathrm{~Hz}$. Continuous EEG data were separated into $1400 \mathrm{~ms}$ epochs, commencing $200 \mathrm{~ms}$ prior to stimulus onset taken as baseline. After correction, the average number of trials and the respective ranges in incongruent and congruent color words, color-word associates, and homophones were 99 (71-119), 101 (78-119), 103 (83-120), 101 (82-120), 101 (81-119), and 102 (75-120), respectively.

\section{Procedure}

Participants were instructed to press one of four keys on a computer keyboard with two fingers on each hand for the corresponding color (red, green, yellow and blue) as fast and accurately as possible. The correspondence between colors and keys was balanced across participants.

All 1128 trials were separated into two blocks in a pseudorandom order. The experiment was preceded by four blocks of 20 practice trials. The error rate of each participant was under $10 \%$ after performing these practice trials. Stimuli were presented on a color monitor, and participants were seated about $80 \mathrm{~cm}$ in front of the screen. The vertical and horizontal visual angles were 1.83 and 1.60 degree, respectively. The presentation of stimuli was signaled by a fixation cross ("+") in the center of the screen for $500 \mathrm{~ms}$. Thereafter, there was a blank screen for $500 \mathrm{~ms}$, then the stimulus appeared at the same location as the fixation cross for maximal $2000 \mathrm{~ms}$ or until the participant pressed any of the designated keys. A blank screen for $500 \mathrm{~ms}$ followed and was replaced by the next fixation cross. The whole experimental session lasted about $1 \mathrm{~h}$.

\section{Data analyses}

Due to the low error rate (lower than $3 \%$ ), only trials with correct responses were used for statistical analyses. Trials with reaction times under $200 \mathrm{~ms}$ or above $2000 \mathrm{~ms}$ were also excluded for analyses. Mean amplitudes in the $400-500 \mathrm{~ms}$ time window were used for statistical analyses of the N450. The electrodes were separated into three regions: anterior (F3, Fz, F4), central (C3, Cz, $\mathrm{C} 4)$, and posterior (P3, Pz, P4), and three levels of a Hemisphere factor from left to right: left (F3, C3, P3), middle ( $F z, C z, P z)$, and right (F4, C4, P4). The Greenhouse-Geisser correction for nonsphericity was used whenever appropriate and epsilon-corrected $P$-values are reported together with uncorrected degrees of freedom.

The difference waves between congruent and incongruent conditions in the 400-500 ms time window for all stimulus types, in the 700-1000 ms time window for color words and in the 600-800 ms time window for homophones and color-word associates (see results), were calculated. The scalp potential topographic maps were generated using a two-dimensional spherical spline interpolation (Perrin et al., 1989) and a radial projection from $\mathrm{Cz}$.

\section{RESULTS}

\section{Behavioral data}

The behavioural data are shown on Table 1. For reasons of integrity the table shows the data for congruent and incongruent trials as well as for neutral trials that served as filler items and did not enter the statistical analyses. An ANOVA for reaction times with factors Congruency (congruent vs. incongruent) and Type (color words vs. colorword associates vs. homophones) revealed a Type effect $(F(2,36)=7.01, P<0.01, \varepsilon=0.84)$, a Congruency effect $(F(1,18)=84$. 32, $P<0.0001)$, as well as a marginally significant interaction $(F(2,36)=3.16, P=0.06, \varepsilon=0.93)$. Type specific $t$-tests revealed reliable Congruency effects for all conditions (one-tailed): color words $(t(18)=7.01, P<$ $0.0001)$, homophones $(t(18)=6.46, P<0.0001)$, and colorword associates $(t(18)=1.88, P<0.05)$.

\section{ERP data}

The N450. ERPs at a midline posterior recording site $(\mathrm{Pz})$ and topographic maps of the difference wave in the three experimental conditions are shown in Fig. 1A. An ANOVA with factors Type (color words vs. color-word associates vs. homophones), Congruency (congruent vs. incongruent), Hemisphere (left vs. middle vs. right) and Region (anterior vs. central vs. posterior) was performed for mean amplitudes in the 400-500 ms time interval. An effect of Congruency $(F(1,18)=10.33, P<0.01)$ and $a$ three-way interaction between Congruency, Type and Region $(F(4,72)=2.81, P<0.05, \varepsilon=0.76)$ were obtained. Following up the latter interaction, type specific analyses of Congruency effects were conducted separately for each region. These analyses revealed significant effects of Congruency for color words all over the scalp (anterior: $F(1,18)=14.05, P<0.01$; central: $F(1,18)=14.34, P<0.01$; posterior: $F(1,18)=13.16, P<0.01)$. For color-word associates significant effects of Congruency were obtained over the posterior region only $(F(1,18)=6.07, P<0.05)$. No effects were obtained for homophones $(F$-values $<1)$.

The late positivity. As apparent from Fig. 1B, starting at around $600 \mathrm{~ms}$ post stimulus, there was a positivity for incongruent homophones and, slightly delayed, for incongruent color words. We performed post hoc contrasts for the mean amplitudes of congruent and incongruent trials in all three conditions in this late time period. This post hoc analysis was restricted to left posterior electrodes (PO3, $\mathrm{PO} 7, \mathrm{P} 3, \mathrm{O} 1)$ at which the late positivity was largest. More positive going ERPs to incongruent homophones were obtained in the $600-800$ ms time window $(F(1,18)=6.86$,

Table 1. Mean reaction times (RT in ms) and standard deviations (in parentheses) for all the stimulus types

\begin{tabular}{|c|c|c|c|c|c|c|c|}
\hline & \multicolumn{2}{|c|}{ Color words } & \multicolumn{2}{|c|}{ Color-word associates } & \multicolumn{2}{|c|}{ Homophones } & \multirow[t]{2}{*}{ Neutrals } \\
\hline & Congr. & Incongr. & Congr. & Incongr. & Congr. & Incongr. & \\
\hline RT & $686(76)$ & 744 (79) & $721(82)$ & $744(83)$ & $685(65)$ & 737 (77) & $708(73)$ \\
\hline
\end{tabular}

Congr., congruent; Incongr., incongruent. 
A. The $\mathrm{N} 450$

cobr werds

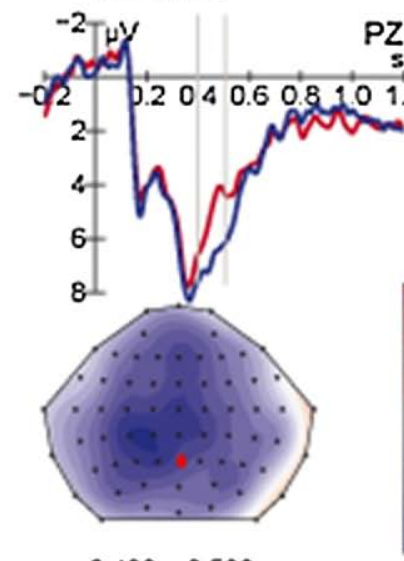

$0.400 . .0 .5008$
$+2.0$

$\mu \mathrm{V}$

$-2.0$

B. The late component

color words

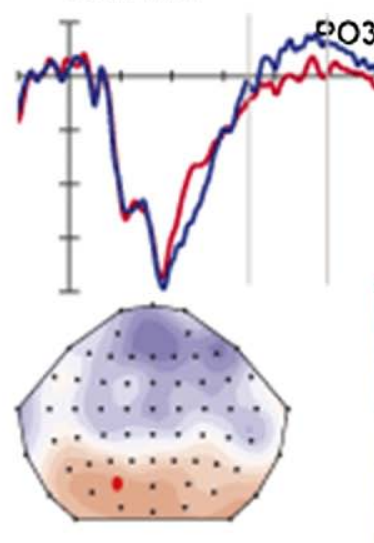

$0.700 . .1 .000=$

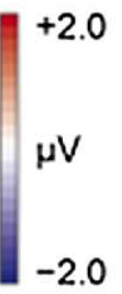

homophoros
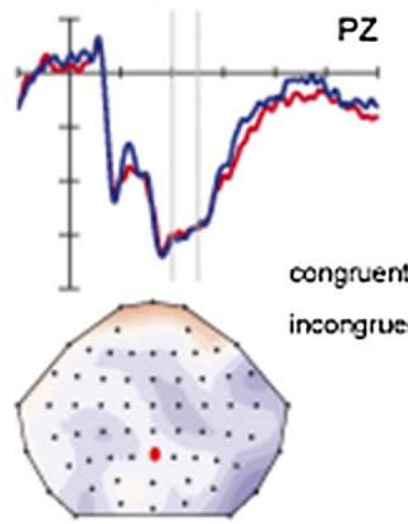

$0.400 . .0 .500 \mathrm{~s}$ color-word associates
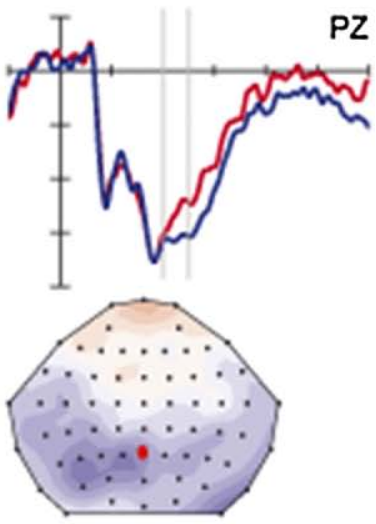

$0.400 . .0 .500 \mathrm{~s}$

Fig. 1. Grand-average ERPs at (A) Pz, and (B) PO3 for incongruent (red line) and congruent conditions (blue line), and topographic maps of the voltage amplitudes for difference waves (incongruent minus congruent) in the 400-500 ms window and in the late time window (incongruent minus congruent) as detected by post hoc contrast. Left: color words. Center: homophones. Right: color-word associates.

$P<0.05)$. For color words a similar effect was obtained in the time window from 700 to $1000 \mathrm{~ms}(F(1,18)=5.91$, $P<0.05)$. No late effects were obtained for color-word associates ( $F$-values $<1$ ) (Fig. 1B).

\section{DISCUSSION}

Previous behavioral studies with Stroop paradigms showed that phonology plays a role in constraining the meaning of the written Chinese word (Chen and Tsoi, 1990; Guo et al., 2005; Saalbach and Stern, 2004; Spinks et al., 2000). In this study, we further studied the role of phonology in semantic access of written Chinese words using a Stroop task with ERP recordings. If phonology plays an important role, we expected to observe similar behavioral and ERP interference effects for color words and for homophones. Besides, we also wanted to explore the sensitivity of the N450 to the amount of semantic conflict with color-word associates. We found behavioral Stroop interference effects for color words, homophones of color words, and color-word associates. In the ERP data, an N450 was observed for incongruent color words and color-word associates, but not for the homophones. In addition, a late positivity with a left posterior scalp topography was found for incongruent color words and homophones. This late effect was absent for color-word associates.

\section{The semantic activation processes for Chinese single-character words}

The behavioral interference effects for color words and homophones were comparable, and confirmed findings from many other previous studies (Chen and Tsoi, 1990; Guo et al., 2005; Saalbach and Stern, 2004; Spinks et al., 2000). The N450 for incongruent color words resembled findings in Stroop tasks in different languages (Liotti et al., 2000; Quu et al., 2006). This N450, however, was absent for incongruent homophones of color words. This finding suggests that incongruent homophones did not induce 
semantic conflict in the Stroop task. If a word could activate the meaning of other words with the same pronunciation, an N450 for incongruent homophones would have been expected. These findings thus compromise the view that phonology plays a dominant role in semantic access in Chinese.

\section{The sensitivity of the N450 to the amount of semantic conflict}

A second is issues addressed in the present study concerns the sensitivity of the N450 to the amount of semantic conflict. We therefore added color-word associates, which due to their lower association strength should induce less semantic conflict than the color words. As in previous studies, the behavioral Stroop effects for color-word associates were less prominent than those for color words but statistically reliable (Klein, 1964; Saalbach and Stern, 2004). In the ERP data, an N450 effect was obtained for incongruent color word associates. This effect was attenuated as compared to the N450 for color words, and was restricted to posterior regions. This attenuated $\mathrm{N} 450$ effect parallels the less pronounced behavioral interference effects for color word associates and suggests that the N450 is sensitive to the amount of the semantic conflict induced by incongruent color words. These findings confirm that the N450 is corresponding to the semantic component of Stroop interference according to the dual-route model of reading.

\section{The source of the behavioral Stroop interference effect for homophones}

An interesting issue concerns the source of the behavioral Stroop interference effect for homophones. In fact, response times to incongruent homophones were about 50 ms longer than for congruent homophones and no N450 effect was obtained for incongruent homophones. A possibility is that this behavioral effect arose at a later stage of lexical processing. In support of this view, we found a late positivity over left posterior regions for incongruent homophones and color words, for which pronounced behavioral interference effects were obtained. The late positivity has high resemblance with an ERP effect recently reported by Liotti et al. (2000). Using English color words they found a late positivity to incongruent color words. On the basis of its left posterior topography and dipole modeling conducted with similar waveforms (Abdullaev and Posner, 1998), Liotti and colleagues take the late positivity to reflect re-entrant activation of word-processing regions. The late positivity for homophones could possibly mean that at this late stage incongruent homophones activated the meaning of the incongruent color words with the same pronunciations. Therefore, it is reasonable to assume that the late positivity reflects the additional processing of the meaning of the incongruent color words in case of conflicting phonological information carried by incongruent color words and homophones. As only incongruent color words and homophone, two conditions which showed strong behavioral interference effects and also had incongruent phonology to colors to be named, elicited this late positivity, it might be associated with processing mechanisms of the phonological component of Stroop interference according to the dual-route model.

\section{CONCLUSION}

The present results do not support the view of a strong role of phonology in accessing meaning of visual Chinese single-character words. It is more likely that semantic access in Chinese language relies heavily on the orthographysemantic route. A possible limitation of our approach could be that due to the constraints of the Stroop task, only a small set of stimuli could be used. With a small set of homophones, it is impossible to distinguish for example between characters for which the phonetic part contributes to pronunciation and those for which it does not. Additionally, each stimulus was repeated many times, which could influence the ecological validity of the results. Thus, additional studies with different experimental paradigms (e.g., lexical decision tasks) are needed to further disentangle the role of phonology in semantic access of written Chinese words.

Acknowledgments-The first author was financially supported by Deutscher Akademischer Austausch Dienst under "DAAD Sandwich Scholarship." The authors acknowledge Stefanie König, Anne Lohs, Julia Hoffmann, Kristina Lemke, Hauke Trustorff, Mauricio Parra for their kind help with data collection, as well as Martina Becker, Nicola Ferdinand, Bertram Opitz for their patient instruction for program compiling and EEG data pre-processing. Thanks are specially given to Professor Raymond C K Chan for helpful comments on an early version of this manuscript and also for his kind help with English writing.

\section{REFERENCES}

Abdullaev YG, Posner MI (1998) Event-related brain potential imaging of semantic encoding during processing single words. Neuroimage 7(1):1-13.

Bakan P, Alperson B (1967) Pronounceability, attensity, and interference in the color-word test. Am J Psychol 80(3):416-420.

Chen HC, Shu H (2001) Lexical activation during the recognition of Chinese characters: evidence against early phonological activation. Psychon Bull Rev 8(3):511-518.

Chen HC, Tsoi KC (1990) Symbol-word interference in Chinese and English. Acta Psychol 75:123-138.

Coltheart M, Rastle K, Perry C, Langdon R, Ziegler J (2001) DRC: A dual route cascaded model of visual word recognition and reading aloud. Psychol Rev 108(1):204-256.

Coltheart M, Woollams A, Kinoshita S, Perry C (1999) A positionsensitive Stroop effect: further evidence for a left-to-right component in print-to-speech conversion. Psychon Bull Rev 6(3):456463.

Frost R (1998) Toward a strong phonological theory of visual word recognition: true issues and false trails. Psychol Bull 123(1):71-99.

Guo T, Peng D, Liu Y (2005) The role of phonological activation in the visual semantic retrieval of Chinese characters. Cognition 98(2): B21-B34.

Klein GS (1964) Semantic power measured through the interference of words with color-naming. Am J Psychol 77:576-588.

Liotti M, Woldorff MG, Perez R, Mayberg HS (2000) An ERP study of the temporal course of the Stroop color-word interference effect. Neuropsychologia 38(5):701-711. 
Meng X, Jian J, Shu H, Tian X, Zhou X (2008) ERP correlates of the development of orthographical and phonological processing during Chinese sentence reading. Brain Res 1219:91-102.

Perfetti CA, Zhang S (1995) Very early phonological activation in Chinese reading. J Exp Psychol Learn Mem Cogn 21(1):24-33.

Perrin F, Pernier J, Bertrand O, Echallier JF (1989) Spherical splines for scalp potential and current density mapping. Electroencephalogr Clin Neurophysiol 72:184-187.

Qiu J, Luo YJ, Wang QH, Zhang FH, Zhang QL (2006) Brain mechanism of Stroop interference effect in Chinese characters. Brain Res 1072(1):186-193.

Saalbach H, Stern E (2004) Differences between Chinese morphosyllabic and German alphabetic readers in the Stroop interference effect. Psychon Bull Rev 11(4):709-715.
Spinks JA, Liu Y, Perfetti CA, Tan LH (2000) Reading Chinese characters for meaning: the role of phonological information. Cognition 76(1):B1-B11.

Stroop JR (1935) Studies of interference in serial verbal reactions. J Exp Psychol 18(6):643-662.

Tan LH, Perfetti CA (1997) Visual Chinese character recognition: does phonological information mediate access to meaning? J Mem Lang 37(1):41-57.

Wong KFE, Chen HC (1999) Orthographic and phonological processing in reading Chinese text: evidence from eye fixations. Lang Cogn Process 14(5-6):461-480.

Xu Y, Pollatsek A, Potter MC (1999) The activation of phonology during silent Chinese word reading. J Exp Psychol Learn Mem Cogn 25(4):838-857. 